\title{
HIGHER ORDER STATISTICS OF THE DISCRETE WIENER MODEL FOR APPLICATION IN NONLINEAR PROCESS MODELING AND IDENTIFICATION
}

\author{
Atalla I. Hashad \\ Charles W. Therrien \\ Department of Electrical and Computer Engineering \\ Naval Postgraduate School, Monterey, CA 93943-5000 \\ e-mail: therrieneece.nps.navy.mil
}

\begin{abstract}
The discrete Wiener model of nonlinear systems is used to represent discrete stochastic processes. The higher order statistics of the model output are analyzed and shown to have properties that greatly reduce the complexity of their computation. An efficient and structured procedure of computing the cumulants of the output of this model is described. Finally, an example of applying these results in nonlinear system identification and random process modeling is presented.
\end{abstract}

\section{INTRODUCTION}

A stationary discrete random process described by a set of statistics can be modeled as the output of a system driven by another random process of known statistics. The modeling is complete when the system parameters are successfully identified such that the model output statistics match those of the given process according to some criterion. When a process is Gaussian it can be efficiently modeled as the output of a linear system driven by white Gaussian noise. The system parameters, generally the ARMA coeficients, can be obtained by matching the second-order statistics of the modeled process with those of the model output. In addition a significant amount of work has been done for modeling a non-Gaussian process as the output of a linear system driven by a white non-Gaussian process of known higher-order statistics $[1,2,3]$. In this case, a set of equations defining the model output cumulants (polyspectra) as functions of the system impulse response (transfer function) is formulated from which the model MA and AR parameters can be obtained.

It is well recognized however, that linear models are not capable of modeling a wide class of processes $[4,9,10]$. In this case a nonlinear mechanism must be considered to describe the given process. A discrete nonlinear system driven by a white Gaussian process is one of the mechansims that generate a discrete non-Gaussian process. If the system is describable by a set of parameters that can also define the input-output relations then the output statistics can be related to the input statistics using these parameters. Since the input is white Gaussian the output statistics of any order are completely determined by the input statistics up to the second order only. This system, which is analogous to the innovations representation for Gaussian processes, can be used to generate a large class of processes with continuous polyspectra. It is not suitable however for representing processes with periodicities or impulses in the polyspectra.

The remainder of this paper is organized as follows. In Section 2 we review the Wiener model of discrete nonlinear systems which is the mechanism we apply to model a discrete non-Gaussian process. In Section 3 the cumulants of the Wiener model output are formulated and an organized procedure for computing these cumulants is described. In Section 4 an example of modeling a discrete non-Gaussian process as the output of a Wiener nonlinear model with different dimensions shows the effect of the model parameters on the modeling error.

\section{THE WIENER MODEL OF DISCRETE NONLINEAR SYSTEMS}

The output $x(n)$ of a well behaved nonlinear system driven by an input $w(n)$ can be described using the Volterra representation [5]

$$
x(n)=\mathcal{H}_{0}[w(n)]+\mathcal{H}_{1}[w(n)]+\cdots+\mathcal{H}_{p}[w(n)]+\cdots
$$

where the $\mathcal{H}_{i}[w(n)]$ is the $i^{\text {th }}$ order homogeneous Volterra functional of the form

$$
\begin{aligned}
& \mathcal{H}_{i}[w(n)] \\
& =\sum_{k_{1}=0}^{\infty} \cdots \sum_{k_{i}=0}^{\infty} h_{i}\left(k_{1}, k_{2}, \cdots, k_{i}\right) w\left(n-k_{1}\right) \cdots w\left(n-k_{i}\right)
\end{aligned}
$$

and where $h_{i}\left(k_{1}, k_{2}, \cdots, k_{i}\right)$ is known as the kernel of the functional. In this representation the magnitude of the input must be restricted to regions that ensure the convergence of this Taylor series-like representation. The individual kernels $h_{i}$ cannot be identified unless the representation is assumed to consist of single functional $\mathcal{H}_{i}[w(n)]$ [5]. To circumvent these limitations Wiener derived a set of nonhomogeneous functionals called G-functionals which are orthogonal when the input $w(n)$ is white Gaussian [6]. In this representation

$$
x(n)=\mathcal{G}_{0}+\mathcal{G}_{1}[w(n)]+\mathcal{G}_{2}[w(n)]+\cdots+\mathcal{G}_{p}[w(n)]+\cdots
$$


where

$$
\begin{gathered}
\mathcal{G}_{0}=g_{0} \\
\mathcal{G}_{1}=\sum_{n=0}^{\infty} g_{1}(k) w(n-k) \\
\mathcal{G}_{2}=\sum_{h_{1}=0}^{\infty} \sum_{k_{2}=0}^{\infty} g_{2}\left(k_{1}, k_{2}\right) w\left(n-k_{1}\right) w\left(n-k_{2}\right)+g_{0(2)} \\
\vdots
\end{gathered}
$$

In general $\mathcal{G}_{p}$ is a sum of homogeneous functionals of order $p$ and lower such that for any homogeneous functional $\mathcal{F}_{m}[w(n)]$ with $m<p$,

$$
\mathcal{E}\left\{\mathcal{F}_{\boldsymbol{m}}[w(n)] \mathcal{G}_{\mathbf{p}}[w(n)]\right\}=0
$$

In previous work [7] we noted that this representation can be realized by a canonical structure that consists of three cascaded sections. The first section is comprised of a bank of linear filters, generally of infinite length, driven by the same sero-mean white Gaussian input $w(n)$ having variance $\sigma_{0}^{2}$. The impulse responses of these filters are given by a complete set of orthonormal functions known as the discrete Laguerre functions with transfer functions

$$
\Lambda_{i}(z)=\sqrt{1-\rho^{2}} \frac{1}{1-\rho z^{-1}}\left[\frac{\rho-z^{-1}}{1-\rho z^{-1}}\right]^{i}
$$

from which

$$
\lambda_{0}(n)=\sqrt{1-\rho^{2}} \rho^{n} u(n)
$$

where $u(n)$ is the discrete unit step function. The remaining impulse response functions can be derived using the recursion relation

$$
\lambda_{k+1}(l)=\rho \lambda_{k+1}(l-1)+\rho \lambda_{k}(l)-\lambda_{k}(l-1)
$$

The second section of this representation consists of identical single-input multi-output memoryless nonlinear blocks. Each of the outputs of the first section is the input of one of these blocks. The input-output relation of the nonlinear block is determined by the set of orthogonal Hermite polynomials

$$
H_{j}(y)=\sum_{m=0}^{\left\lfloor\frac{i}{2}\right\rfloor} \frac{(-1)^{m} j !}{m !(j-2 m) !}\left(\frac{\sigma_{o}^{2}}{2}\right)^{m} y^{j-2 m}
$$

where $\sigma_{0}^{2}$ is the variance of the input $w(n)$. The third section is a multi-input single output stage that forms the output of the model as a weighted sum of products of the outputs of the nonlinear section. Wiener defined a class of nonlinear systems that can have this canonical representation [6]. The output of a system that is considered a member of this class must have a finite variance for a finite variance input. Also the output of this system must become asymptotically independent of the input. In this case the linear section of this model can be approximated

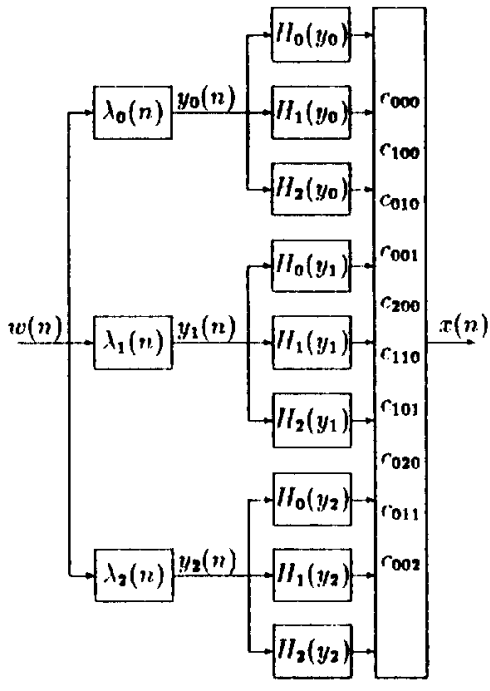

Figure 1. The Wiener model for $N_{L}=2$ and $N_{N}=2$

by a bank of filters that has a finite length $N_{L}$ which corresponds to the highest order of the Laguerre transfer functions. Then the linear section consists of $N_{L}+1$ linear filters with impulse responses $\lambda_{0}(n), \lambda_{1}(n), \cdots, \lambda_{N_{2}}(n)$ and outputs $y_{0}(n), y_{1}(n), \cdots, y_{N_{L}}(n)$ while the nonlinear section consists of Hermite polynomials up to order $N_{N}$ which represents the highest degree of nonlinearity in the representation (3). Fig. 1 depicts the detailed Wiener model for $N_{L}=2$ and $N_{N}=2$.

When the system has finite dimensions $N_{L}$ and $N_{N}$, the output is the weighted sum of a set of multinomials denoted by $Q \alpha$ and defined by the vector of indices

$$
\alpha=\left[\alpha_{0}, \alpha_{1}, \alpha_{2}, \cdots, \alpha_{N_{L}}\right]
$$

such that

$$
Q_{\alpha}(n)=H_{\alpha_{0}}\left(y_{0}(n)\right) H_{\alpha_{1}}\left(y_{1}(n)\right) \cdots H_{\alpha_{N_{L}}}\left(y_{N_{L}}(n)\right)
$$

The product of the Hermite polynomials to form $Q_{\alpha}$ is such that one and only one Hermite polynomial from each nonlinear block is taken at a time and the sum of their orders does not exceed $N_{L}$. Then the output $x(n)$ is given by

$$
x(n)=\sum_{\sum \alpha \leq N_{L}} \operatorname{co} Q_{\alpha}(n)
$$

where the vector sum in the subscript means the sum of the components of the vector $\alpha$. The coefficients $c_{\alpha}$ are the system parameters included in the third section.

\section{CUMULANTS OF THE OUTPUT OF THE DISCRETE WIENER MODEL}

The cumulants of the model output $x(n)$ are obtained by substituting (12) in the cumulant expressions. Since the cumulants are additive in their arguments and have linearity properties [8]; the $k^{\text {th }}$ order cumulant of $x(n)$ is given 
where

$$
\begin{aligned}
& \mathcal{G}_{0}=g_{0} \\
& \mathcal{G}_{1}=\sum_{n=0}^{\infty} g_{1}(k) w(n-k) \\
& \mathcal{G}_{2}=\sum_{k_{1}=0}^{\infty} \sum_{k_{2}=0}^{\infty} g_{2}\left(k_{1}, k_{2}\right) w\left(n-k_{1}\right) w\left(n-k_{2}\right)+g_{0(2)}
\end{aligned}
$$

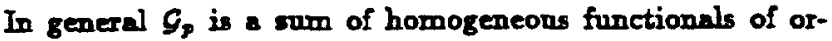
der $p$ and lower wech that for any homogeneous funetional $\mathcal{F}_{m}[\boldsymbol{m}(\boldsymbol{n})]$ with $m<p$,

$$
\mathcal{E}\left\{\mathcal{F}_{m}[\boldsymbol{w}(n)] \mathcal{G}_{s}[\boldsymbol{w}(n)]\right\}=0
$$

In previons work [7] we noted that this representation can be realised by a canonical structure that consists of three cascaded sections. The first section is comprised of a bank of linenr filters, generally of infinite length, driven by the same sero-mean white Gausian inpat $w(n)$ having variance $\sigma_{0}^{2}$. The impulse responses of these filters are given by a complete set of orthonormal functions known as the discrete Laguerre functions with tranefer functions

$$
\Lambda_{i}(x)=\sqrt{1-p^{2}} \frac{1}{1-p z^{-1}}\left[\frac{\rho-z^{-1}}{1-\rho z^{-1}}\right]^{i}
$$

from which

$$
\lambda_{0}(n)=\sqrt{1-p^{2}} p^{n} u(n)
$$

where $u(r)$ is the discrete anit step function. The remsining impulse response functions can be derived using the recuraion relation

$$
\lambda_{k+1}(l)=\rho \lambda_{k+1}(l-1)+\rho \lambda_{k}(l)-\lambda_{k}(l-1)
$$

The recond section of this representation consists of identical single-input multi-output memoryless nonlinear blocks. Each of the outputs of the firat section is the input of one of these blociss. The input-output relation of the nonlinear block is determined by the set of orthogonal Hermite polynominis

$$
H_{j}(y)=\sum_{m=0}^{\left\lfloor\frac{1 j\rfloor}{j}\right\rfloor} \frac{(-1)^{m} j !}{m !(j-2 m) !}\left(\frac{\sigma_{0}^{2}}{2}\right)^{m} y^{j-2 m}
$$

Where $\sigma_{0}^{2}$ is the varinnce of the input $20(n)$. The third section is a multi-input single ontpnt stage that forms the ontpat of the model as a weighted sum of prodncts of the outputs of the nonlinear section. Wiener defined a class of nonlinear systems that can have this canonical representation [6]. The outpat of a system that is considered member of this class mast have a finite variance for a finite varinnce input. Also the output of this system must become asymptotically independent of the input. In this case the linear section of this model can be approximated

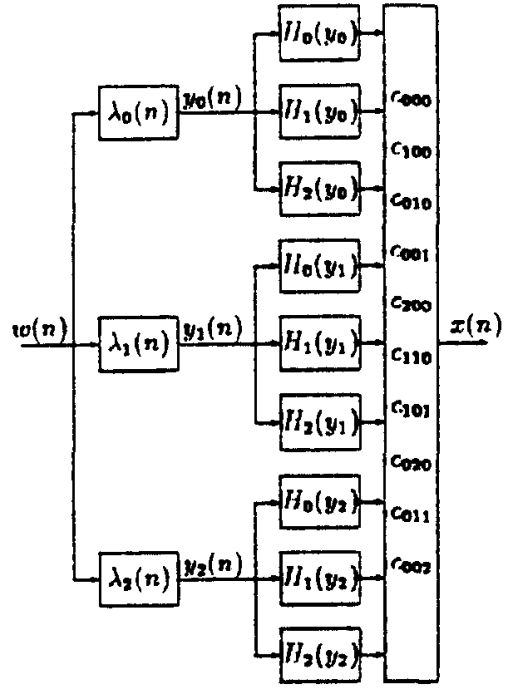

Figure 1. The Wiener model for $N_{L}=2$ and $N_{N}=2$

by a bank of filters that has a finite length $N_{L}$ which corresponds to the highest order of the Laguerre transfer functions. Then the linear section consists of $N_{L}+1$ linear filters with impulse respones $\lambda_{0}(n), \lambda_{1}(n), \cdots, \lambda_{N_{2}}(n)$ and outputs $y_{0}(n), y_{1}(n), \cdots, y_{N_{L}}(n)$ while the nonlinear section consists of Hermite polynomials up to order $N_{N}$ which represents the highest degree of nonlinearity in the representation (3). Fig. I depicts the detailed Wiener model for $N_{L}=2$ and $N_{N}=2$.

When the system has finite dimensions $N_{2}$ and $N_{N}$, the outpat is the weighted sum of a set of multinomials denoted by $Q_{\alpha}$ and defined by the vector of indices

$$
\alpha=\left[\alpha_{0}, \alpha_{1}, \alpha_{2}, \cdots, \alpha_{N_{L}}\right]
$$

such that

$$
Q_{\alpha}(n)=H_{a_{0}}\left(y_{0}(n)\right) H_{a_{2}}\left(y_{1}(n)\right) \cdots H_{\alpha_{N_{2}}}\left(y_{N_{2}}(n)\right)
$$

The product of the Bermite polynomials to form $Q_{a}$ is such that one and only one Bermite polynomial from each nonlinear block is taken at a time and the sam of their orders does not exceed $N_{L}$. Then the outpnt $x(n)$ is given by :

$$
x(n)=\sum_{\sum \alpha \leq N_{L}} \operatorname{co} Q \alpha(n)
$$

where the vector som in the subecript means the sam of the components of the vector $\alpha$. The coefficients $c \alpha$ are the syatem parameters included in the third section.

\section{CUMULANTS OF THE OUTPUT OF THE DISCRETE WIENER MODEL}

The comalants of the model output $x(n)$ are obtained by substituting (12) in the cumulant exprearions. Since the cumulants are additive in their arguments and have linenrity properties [8]; the $k^{\text {th }}$ order cumulant of $x(n)$ is given 
by

$$
\begin{aligned}
& C_{m}^{(h)}\left(l_{1}, l_{2}, \cdots, l_{h-1}\right) \\
& =\sum_{a_{1}} \cdots \sum_{\alpha_{h}} c_{\alpha_{1}} \cdots c_{a_{k}} C_{a_{1}, \cdots, a_{k}}^{(k)}\left(l_{1}, l_{2}, \cdots, l_{k-1}\right)
\end{aligned}
$$

where

$$
\begin{aligned}
& C_{a_{1}, a_{2}, \cdots, a_{k}}^{(k)}\left(l_{1}, l_{2}, \cdots, l_{k-1}\right) \\
& =\operatorname{cum}\left(Q_{a_{0}}(n) Q_{a_{1}}\left(n+l_{1}\right) \cdots Q_{a_{k}}\left(n+l_{k-1}\right)\right.
\end{aligned}
$$

Since the $Q$-polynomials have zero mean except $Q_{0}$ which equals one, it follows that

$$
C_{\approx}^{(1)}=\mathcal{E}\{(x(n))\}=c_{00} \ldots 00
$$

This means that when identifying the model parameters to match the cumulants of a given random process the coefficient $c_{00 \ldots 00}$, is directly determined by taking the expectation of the process. Since the cumulants do not change when a constant value is added to or subtracted from the process $x(n)$ we can assume that the model output has gero mean , i.e., $c_{00} \ldots 00=0$ to save a significant amount of computations and exclude the terms that have this constant value as a multiplier from the cumulant expressions.

The cross-cumulants of the Q-polynomials of orders 2, 3 and 4 are given by

$$
\begin{aligned}
& C_{\alpha_{1}, a_{2}}^{(2)}(l)=\mathcal{E}\left\{Q_{\alpha_{1}}(n) Q_{a_{2}}(n+l)\right\} \\
& C_{a_{1}, a_{2}, a_{3}}^{(3)}\left(l_{1}, l_{2}\right)=\mathcal{E}\left\{Q_{\alpha_{3}}(n) Q_{a_{2}}\left(n+l_{1}\right) Q_{a_{3}}\left(n+l_{2}\right)\right\} \\
& C_{a_{1}, a_{2}, a_{3}, a_{4}}^{(4)}\left(l_{1}, l_{2}, l_{3}\right) \\
& =\mathcal{E}\left\{Q_{a_{1}}(n) Q_{a_{3}}\left(n+l_{1}\right) Q_{a_{3}}\left(n+l_{2}\right) Q_{\alpha_{4}}\left(n+l_{2} 3\right)\right\} \\
& -\mathcal{E}\left\{Q_{\alpha_{1}}(n) Q_{\alpha_{2}}(n+l)\right\} \mathcal{E}\left\{Q_{\alpha_{3}}\left(n+l_{2}\right) Q_{\alpha_{1}}\left(n+l_{3}\right)\right\} \\
& -\mathcal{E}\left\{Q_{\alpha_{1}}(n) Q_{\alpha_{3}}\left(n+l_{2}\right)\right\} \mathcal{E}\left\{Q_{a_{2}}\left(n+l_{1}\right) Q_{\alpha_{4}}\left(n+l_{3}\right)\right\} \\
& -\mathcal{E}\left\{Q_{a_{1}}(n) Q_{a_{4}}\left(n+l_{3}\right)\right\} \mathcal{E}\left\{Q_{a_{3}}\left(n+l_{1}\right) Q_{a_{3}}\left(n+l_{2}\right)\right\}
\end{aligned}
$$

Thus to compute the cumulant of the model output it is required to compute the cross-moments of the Q-polynomials. Since these are products of polynomials of the outputs of the linear filters which are driven by a gero-mean white Gaussian process, the cross-moment of Q-polynomials is a sum of terms of the form

$$
\mathcal{E}\left\{y_{0}^{\nu_{10}}(n) y_{1}^{\nu_{11}}(n) \cdots y_{0}^{\nu_{20}}(n+l 1) \cdots \cdots y_{N L}^{\nu_{k N_{L}}}\left(n+l_{k-1}\right)\right\}
$$

Where the $y_{i}$ are zero-mean Gaussian random variables with variance $\sigma_{0}^{2}$. The $\nu_{i j}$ are non-negative integers that correspond to the exponents of the $y_{i}$ when substituting (9) and (11) in (16). The value of this expectation is zero if the sum of the exponents of the random variables is odd. Otherwise, the value of this expectation is the sum of products of expectations of all possible pairing permutations. In each case the expectation of pairs of these random variables is given by

$$
R_{y_{i} y_{j}}(l)=\mathcal{E}\left\{y_{i}(n) y_{j}(n+l)\right\}=\sigma_{0}^{2} r_{j-i}(l)
$$

where $\boldsymbol{r}_{j-i}(l)$ is the Laguerre cross-correlation sequence

$$
\boldsymbol{r}_{j-i}(l)=\sum_{k=0}^{\infty} \lambda_{i}(k) \lambda_{j}(l+k)
$$

which is easy to compute and tabulate [T]. Except for $r_{0}(I)$, which is given by

$$
r_{0}(l)=\rho^{|z|}
$$

these sequences are strictly single-sided, i.e.,

$$
r_{d}(l)=0 \quad \text { for } d>0 \text { and } l \leq 0
$$

and satisfy

$$
\boldsymbol{r}_{-d}(l)=\boldsymbol{r}_{d}(-l)
$$

Therefore any permutation of the expression (17) that includes $r_{d}(-l)$ or $r_{-d}(l)$ is identically zero. In addition, when expanding the Q-polynomials in (16) using (9) and (11) many of the terms have a common factor that sums to zero. In fact we have shown [11] that only the product of the first term of each of the Hermite polynomials is required because all the other terms cancel out. The computation of cross-moment of the Q-polynomials in (16) reduces to just computing

$$
\mathcal{E}_{H}\left\{y_{0}^{\alpha_{10}}(n) y_{1}^{\alpha_{11}}(n) \cdots y_{0}^{\alpha_{20}}(n+l 1) \cdots \cdots y_{N L}^{\alpha_{k N}}\left(n+l_{k-1}\right)\right\}
$$

where the operator $\mathcal{E}_{H}$ means that the expectation is taken by summing all the values of permutations excluding those that have the quantity $r_{0}(0)$ in the product.

From these properties the Q-polynomial cross-moment can be computed from the set vectors of indices $\alpha_{i}$. First the necessary and sufficient conditions for these moments not to be identically zero are set up. A necessary condition is that the sum of all the components in the vectors $\alpha_{i}$ must be even. Sufficient conditions can be specified as follows. Let the vector of indices $\alpha_{i}$ in (10) be partitioned as

$$
\alpha=\left[\begin{array}{ll}
\gamma_{N_{L}+1-m} & \beta_{m}
\end{array}\right]
$$

such that $\beta_{m}$ represents the $m$ components of the vector that are the exponents of the outputs of the Laguerre filters with highest orders, and $\gamma_{N_{L}+1-m}$ represents the $N_{L}+1-m$ outputs of the filters with lowest Laguerre orders. Further, let us define the vector notation $\sum \alpha$ to mean the sum of all the components in the vector $\alpha$. The sufficient conditions for the Q-polynomial cross-moment not to be identically zero depends upon the order of the moment. For the second order moment $\mathcal{E}\left\{Q_{\alpha}(1)(n) Q_{\alpha}(a)(n+l)\right\}$ with $l \geq 0$ it is sufficient to have

$$
\begin{aligned}
& \text { - } \sum \alpha^{(1)}=\sum \alpha^{(2)} \\
& \text { - } \sum \beta_{M}^{(1)} \leq \beta_{m}^{(2)} \text { for } m=0,1,2, \cdots, N_{L}-1
\end{aligned}
$$

to guarantee that the moment is not identically sero. The value of the moment is then obtained by using the method described in [7]. The equivalent conditions for the third order moment $\mathcal{E}\left\{Q_{\alpha(1)}(n) Q_{\alpha(j)}\left(n+l_{1}\right) Q_{\alpha(3)}\left(n+l_{2}\right)\right\}$ for $l_{2} \geq l_{1} \geq 0$ are

$$
\text { - The sum } \sum \alpha^{(1)}+\sum \alpha^{(2)}+\sum \alpha^{(3)} \text { is even. }
$$


- The sum of the components of any two vectors is greater than or equal to the sum of the components in the third, i.e.,

$$
\begin{aligned}
\sum \alpha^{(1)}+\sum \alpha^{(2)} & \geq \sum \alpha^{(1)} \\
\sum \alpha^{(1)}+\sum \alpha^{(3)} & \geq \sum \alpha^{(2)} \\
\sum \alpha^{(2)}+\sum \alpha^{(3)} & \geq \sum \alpha^{(1)}
\end{aligned}
$$

- The sub vectors $\beta_{m}$ and $\gamma_{N_{L}+1-m}$ for $m=0,1, \cdots, N_{L}$ of the three vectors satisfy the relations

$$
\begin{aligned}
& \beta_{m}^{(1)} \leq \beta_{m}^{(2)}+\beta_{m}^{(3)} \\
& \beta_{m}^{(2)} \leq \sum \alpha^{(1)}+\beta_{m}^{(3)} \\
& \gamma_{m}^{(3)} \leq \gamma_{m}^{(1)}+\gamma_{m}^{(2)}
\end{aligned}
$$

Similar relations between the vectors of indices $\alpha_{i}$ can be derived such that a $Q$-polynomial cross-moment of any order is not identically sero. Then the cumulants of the model output $x(n)$ are computed in steps as follows:

- From the model dimensions $N_{L}$ and $N_{N}$ the set of the vectors of indices $\alpha_{i}$ is constructed such that each vector consists of $N_{L}+1$ components. Each component assumes the values $0,1, \cdots, N_{N}$ such that the sum of the components in each vector does not exceed $N_{N}$.

- For the $k^{\text {th }}$ order cumulant all the combinations of $k$ vectors of indices is checked to see if the corresponding term is identically zero. If it is not the value of the corresponding Q-polynomial is computed and substituted in the cumulant expression.

Since the number of Q-polynomials in (12) increases proportional to the value of $N_{L} \times N_{N}$ the number of terms in the expression (13) increases as $\left(N_{L} \times N_{N}\right)^{k}$. Thus the complexity of the computation increases very rapidly with model dimensions and increases exponentialy with cumulant order. Fortunately, the number of the terms that are identically zero also increases rapidly with the increase of $N_{L}$ and $N_{N}$ such that the ratio of the number of zero terms to the total number of terms increases and maintains the efficiency of the Wiener model in representing discrete nonlinear processes. For example the second-order cumulant of the output of the model in Fig. (1) is given by

$$
C_{\mathrm{z}}^{(2)}(l)=\mathrm{c}^{T} \mathbf{R}^{(2)} \mathbf{c}
$$

where $c$ is the vector of coefficients in the third section of the model and $\mathbf{R}^{(2)}$ is a block diagonal matrix with upper triangular diagonal blocks. The total number of the matrix elements is 81 of which 54 elements are identically zero. The third order cumulant is given by

$$
C_{\mathrm{z}}^{(3)}\left(l_{1}, l_{2}\right)=\mathrm{c}^{T} \mathbf{R}^{(3)} \mathbf{c} \otimes \mathbf{c}
$$

where $\mathbf{c} \otimes \mathbf{c}$ is the kronecker product of the vectors of coeffecients and $\mathbf{R}^{(3)}$ is the matrix of cross-moments of size $81 \times 9$ that has 215 elements which are identically zero. Table (1) shows the ratio of the non-zero elements to the total number of elements in $\mathbf{R}^{(3)}$ for different model dimensions.

\begin{tabular}{|c|c|c|c|}
\hline$N_{L} / N_{N}$ & 2 & 3 & 4 \\
\hline 1 & .3360 & .3086 & .3003 \\
\hline 2 & .2922 & .2524 & .2560 \\
\hline 3 & .2766 & .2225 & .0266 \\
\hline 4 & .2714 & & \\
\hline 5 & .2706 & & \\
\hline
\end{tabular}

Table 1. The ratio of the non-zero terms in the third-order cumulant function

\section{SIMULATION RESULTS}

To demonstrate the efficiency of the Wiener model in modeling stochastic processes with continuous polyspectra we present the results of one example. This example uses a sequence of measurement obtained from data set $B$ of the Santa Fe Institue sets of data [12]. This data represents the measurement of the blood oxygen content recorded from a patient in the sleep laboratory of the Beth Israel Hospital in Boston Massachusetts. This sequence, shown in Fig. 2 which corresponds to the measurement when the patient was in the awake state, consists of 2500 points. The second and third order cumulants of this sequence were estimated. Three models of sixes $\left[N_{L}=1, N_{N}=2\right],\left[N_{L}=1, N_{N}=3\right]$ and $\left[N_{L}=1, N_{N}=2\right]$ were used to model the data. In each case a number of samples of the covariance function, or the third-order cumulant, or both were chosen and the model parameters $\left(\rho\right.$ and $c_{\alpha}$ ) were adjusted to best match these samples in a mean-square sense. The theoretical expressions, developed above, were used to compute the model cumulants and readjust the parameters. The optimization was carried out by a recursive least squares technique similar to that employed in the Extended Kalman Filter application in solving a set of nonlinear equations [13]. The value of the parameters were then used to build a model which was driven with white noise to produce simulated data. Cumulants for the simulated data were then estimated and compared to those for the original data. The best match according to the mean-square optimization criterion was obtained for the model with $N_{L}=2$ and $N_{N}=2$. The simulated sequences for the three cases are shown in Fig. 3, Fig. 4 and Fig. 5.

\section{CONCLUSION}

The Wiener model of discrete nonlinear systems provides an efficient mechanism to represent non-Gaussian random processes that have continuous polyspectra. This model provides a structured method to represent the higher order statistics of the model output due to the orthogonality that exists among the discrete Laguerre functions representing the linear section of the model, and the Hermite polynomials that represent the nonlinearities in the model. The structure of the model together with the high sparseness of the arrays required to express the cumulants of the model output dramtically reduce the cost of the computation of such problens especially when dealing with high order cumulants. Experiments to build models that simultaneously match the second and higher-order cumulants of a given random process demonstrate the viability of the approach. 


\section{REFERENCES}

[1] Raghuveer, M.R. and S.A. Dianat, "On the Existence of Autoregressive Models for Third-Order Cumulant Matching,"IEEE Thansactionss on Acoustics, Speech and Signal Processing, Vol. 37, No. 12, December 1989.

[2] Giannakis, G.B., "On the Identifiability of NonGaussian ARMA Models Using Cumulants," IEEE Transactions on Automatic Control, Vol. 35, No. 1, January 1991.

[3] Fonollosa, Jose A.R, and J. Vidal, "System Identification Using a Linear Combination of Cumulant Slices,"IEEE Thansactions on Signal Processing, Vol. 41, No. 7, July 1993.

[4] Tong, H.,Nonlinear Time Series, A Dynamical System Approach, Oxford University Press, New York 1990.

[5] Schetsen, M.,The Volterra and Wiener Theories of Nonlinear Systems., John Wiley \& Sons, New York 1980.

[6] Wiener, N., Nonlinear Problems in Random Theory., John Wiley \& Sons, New York 1958.

[7] Therrien, C.W. and A.I. Hashad, "The Discrete Wiener Model for Representation of NonGaussian Stochastic Processes," Proc. of Asilomar Conference on Signals, Systems and Computers, Oct. 31-Nov. 3,1993 Pacific Grove, California.

[8] Mendel, J.M., "Tutorial on Higher-Order Statistics (Spectra) in Signal Processing and System Theory," Proc. of the IEEE, Vol. 79, No. 3,March 1991.

[9] Tekalp, A.M. and A.T. Erdem, "Higher-Order Spectrum Factorisation in One and Two Dimensions with Applications in Signal Modeling and Nonminimum Phase System Identification," IEEE Transactions on Acoustics, Speech, and Signal Processing, Vol. 37, No. 10, October 1989.

[10] Erdem, A.T. and A.M. Tekalp, "On the Measure of the Set of Factorisable Ploynomial Bispectra," IEEE Transactions on Acoustics, Speech, and Signal Processing, Vol. 38, No. 9, September 1990.

[11] Hashad, A.I., “Analysis of Non-Gaussian Processes Using Wiener Model of Discrete Nonlinear Systems," Ph.D. Thesis, Naval Postgraduate School, Monterey, California 1994.

[12] Weigend, A.S. and N.A. Gershenfeld, "Time Series Prediction," Proc. of the NATO Advanced Research on Comparative Time Series Analysis, Santa Fe, New Mexico, May 1992.

[13] Anderson, B.D.O. and J.B. Moore, Optimal Filtering, Prentice-Hall,Inc., Englewood Cliff, New Jersey 1979.

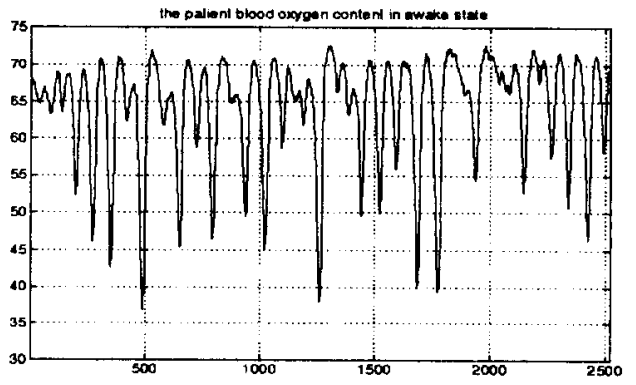

Figure 2. Blood oxygen content data of a patient.

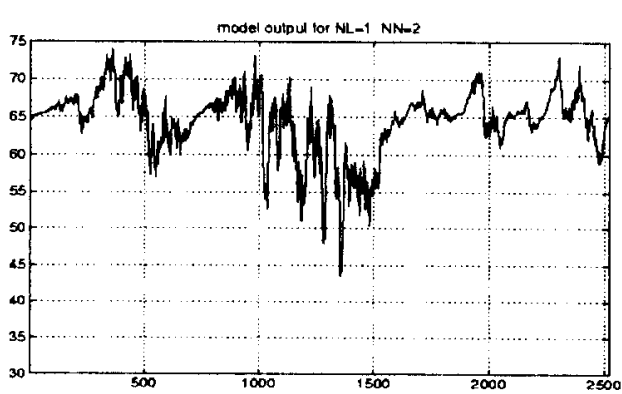

Figure 3. Model output for $N_{L}=1$ and $N_{N}=2$

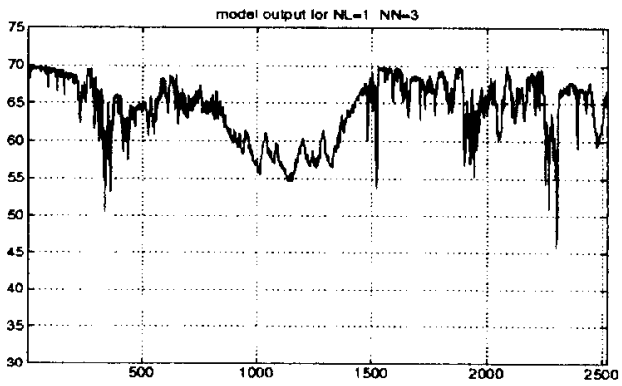

Figure 4. Model output for $N_{L}=1$ and $N_{N}=3$

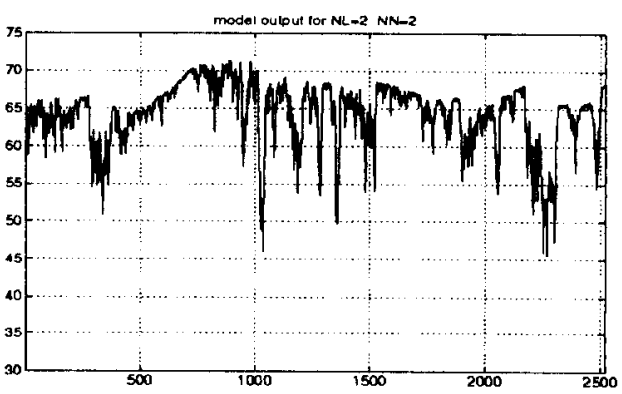

Figure 5. Model output for $N_{L}=2$ and $N_{N}=2$ 